\title{
Reduction of miR-21 induces SK-N-SH cell apoptosis and inhibits proliferation via PTEN/PDCD4
}

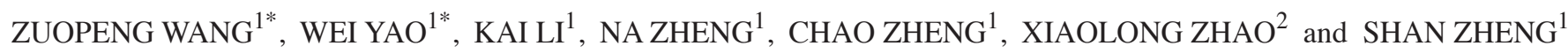 \\ ${ }^{1}$ Department of Pediatric Surgery, Children's Hospital of Fudan University, Shanghai 201102; \\ ${ }^{2}$ Department of Endocrinology, Huashan Hospital of Fudan University, Shanghai 200040, P.R. China
}

Received September 16, 2015; Accepted January 10, 2017

DOI: $10.3892 / \mathrm{ol} .2017 .6052$

\begin{abstract}
MicroRNA ( $\mathrm{miR} / \mathrm{miRNA}$ )-21 is a well-known oncogenic miRNA that is overexpressed in various types of tumors. The tumor-suppressor genes programmed cell death 4 (PDCD4) and phosphatase tensin homologue (PTEN), are targets of miR-21, and are underexpressed in several types of cancer. However, the expression of miR-21 and its target genes in neuroblastoma (NB) remains unclear. In the present study, a miR-21 inhibitor oligonucleotide was transfected into the SK-N-SH cell line, and the expression of miR-21, PTEN and PDCD4 was detected through quantitative polymerase chain reaction analysis. Western blotting was used to examine levels of PTEN, PDCD4 and caspase-3 proteins. The expression of PTEN and PDCD4 in the SK-N-SH cell line transfected with the miR-21 inhibitor was significantly increased compared with untransfected SK-N-SH and negative control-transfected cells. Cell proliferation was inhibited and the apoptotic ratio was significantly increased in miR-21 inhibitor-transfected cells compared with untransfected SK-N-SH and negative control-transfected cells. Western blot analysis revealed a significant increase in caspase-3 expression compared with untransfected SK-N-SH and negative control-transfected cells. The results of the present study indicate that miR-21 may serve an oncogenic role in the cellular processes underlying NB
\end{abstract}

Correspondence to: Professor Kai Li, Department of Pediatric Surgery, Children's Hospital of Fudan University, 399 Wan Yuan Road, Shanghai 201102, P.R. China

E-mail: likai2727@163.com

*Contributed equally

Abbreviations: PDCD4, programmed cell death 4; PTEN, phosphatase and tensin homologue; NB, neuroblastoma; miRs, microRNAs; DMEM, Dulbecco's modified Eagle's medium; FBS, fetal bovine serum; sh, small hairpin; WB, western blot; GFP, green fluorescent protein; $\mathrm{PIP}_{3}$, phosphatidylinositol 3,4,5-triphosphate; Akt, AKT serine/threonine kinase

Key words: neuroblastoma, SK-N-SH, miR-21, lentivirus, phosphatase tensin homologue, programmed cell death 4 development and thus may be a novel therapeutic target for the treatment of patients with NB.

\section{Introduction}

Neuroblastoma (NB) originates from immature sympathetic neural cells and is one of the most common types of solid malignant tumors in children. Nearly half of all NB cases occur in children $<2$ years old, and the prognosis varies widely, with outcomes ranging between spontaneous regression and mortality (1). NB accounts for 7-10\% of childhood malignancies and $\sim 15 \%$ of all childhood cancer-associated mortalities $(2,3)$. Thus, the treatment and management of NB continues to be a challenge faced by physicians and scientists.

MicroRNAs (miRNAs/miRs) are short non-coding RNAs that control gene activity by targeting post-transcriptional expression of specific genes (4). miRNAs serve an important role in the regulation of fundamental cellular processes, including proliferation, migration and differentiation, and are involved in the pathogenesis of NB by acting as oncogenes or tumor-suppressor genes. For example, miR-23a promotes NB cell migration and invasion by targeting the cadherin 1 gene (5). miR-338-3p suppresses NB proliferation, invasion and migration through phosphatidylinositol-3,4,5-trisphosphate $\left(\mathrm{PIP}_{3}\right)$-dependent rac exchange factor 2 (6). miR-362-5p inhibits the proliferation and migration of NB cells by targeting phosphatidylinositol-4-phosphate 3-kinase catalytic subunit type $2 \beta(7)$. miR-145 regulates the gene expression of hypoxia-inducible factor $2 \alpha$, thus inhibiting the growth, invasion, metastasis and angiogenesis of NB cells (8). Although multiple genetic and molecular lesions have been associated with NB tumorigenesis $(6,7)$, the molecular mechanisms regulating NB remain unclear.

miR-21 has been suggested to be oncogenic in multiple types of tumors. Previous studies have demonstrated that the downregulation of miR-21 suppresses tumor growth and invasion in breast, glioma, gastric and colon cancer cells by directly targeting genes for phosphatase tensin homologue (PTEN) and programmed cell death 4 (PDCD4) (9-12). To the best of our knowledge, no previous studies have investigated the role of miR-21 in the NB SK-N-SH cell line, making the present study the first to examine the association between PTEN and PDCD4 expression, and cell apoptosis in SK-N-SH cells transfected with a miR-21 inhibitor. Elucidating the molecular 
mechanisms underlying NB etiopathogenesis may contribute to the identification of suitable therapeutic agents for the treatment of patients with NB.

\section{Materials and methods}

Cell culture. SK-N-SH, SH-SY5Y and BE2C cells (American Type Culture Collection, Manassas, VA, USA) were obtained for use in the present study. LV3-miR-21 inhibitor-transfected SK-N-SH cells are denoted as 1381 cells. The cells were cultured through serial passage in Dulbecco's modified Eagle's medium (DMEM) with 10\% fetal bovine serum (FBS; both from Gibco; Thermo Fisher Scientific, Inc., Waltham, MA, USA), $100 \mathrm{IU} / \mathrm{ml}$ penicillin and $100 \mu \mathrm{g} / \mathrm{ml}$ streptomycin (both from Invitrogen; Thermo Fisher Scientific, Inc.) in a humidified atmosphere with $5 \% \mathrm{CO}_{2}$ at $37^{\circ} \mathrm{C}$. All procedures were performed according to internationally accepted ethical guidelines. The present study was approved by the Institutional Review Board of the Children's Hospital of Fudan University (Shanghai, China).

Plasmid construction, lentivirus packaging and cell infection. A lentiviral vector, $\mathrm{pGLV} 3 / \mathrm{H1} /$ green fluorescent protein (GFP) + Puromycin (pGLV3; Shanghai GenePhama Co., Ltd., Shanghai, China), was used to construct the pGLV3-miR-21 inhibitor plasmid. The miR-21 inhibitor and negative control (NC) oligonucleotides were synthesized by Shanghai GenePhama Co., Ltd. (Table I). The miR-21 small hairpin (sh) DNA double stranded template sequence was synthesized by Huajin Nano Technology Co., Ltd. (Shanghai, China) using miR-21 inhibitor forward (BamHI) and reverse (EcoRI) primers (Fermentas; Thermo Fisher Scientific, Inc.). Subsequently, the miR-21 inhibitor sequence was inserted into the pGLV3 lentivirus plasmid. pGLV3-shDNA-NC was used as a negative control and was constructed using forward (BamHI) and reverse (EcoRI) primers.

The 293T producer cell line (Cell Bank of Type Culture Collection of the Chinese Academy of Sciences, Shanghai, China) was cultured in DMEM with 10\% FBS, $100 \mathrm{U} / \mathrm{ml}$ penicillin and $100 \mu \mathrm{g} / \mathrm{ml}$ streptomycin. Subsequently, 1 day prior to transfection, the cells $\left(5 \times 10^{5} / \mathrm{ml}\right)$ were seeded into a $15-\mathrm{cm}$ dish. pGLV3-miR-21 inhibitor or pGLV3 vectors and packaging plasmids, including pGag/Pol, pRev and pVSV-G (Shanghai GenePhama Co., Ltd.), were co-transfected using RNAi-Mate (Shanghai GenePhama Co.,Ltd.) according to the manufacturer's protocol. The supernatant was collected $72 \mathrm{~h}$ post-transfection, centrifuged $\left(2,200 \times \mathrm{g}\right.$ at $4^{\circ} \mathrm{C}$ for $\left.4 \mathrm{~min}\right)$, passed through a $0.45-\mu \mathrm{m}$ syringe filter and centrifuged again $(50,000 \mathrm{x} \mathrm{g}$ at $4^{\circ} \mathrm{C}$ for $2 \mathrm{~h}$ ). The viral titer was measured according to the expression of GFP following the manufacturer's protocol. The packaged lentiviruses were designated LV3-miR-21 inhibitor and LV3-NC. The sequences of all vectors were verified through sequence analysis.

Reverse transcription-quantitative polymerase chain reaction $(R T-q P C R)$. Total RNA, was isolated from cells using TRIzol ${ }^{\circledR}$ reagent (Invitrogen; Thermo Fisher Scientific, Inc.) according to the manufacturer's protocol. RT was performed using gene-specific RT primers from the TaqMan ${ }^{\circledR}$ MicroRNA Assay kit (Applied Biosystems; Thermo Fisher Scientific, Inc.) and the TaqMan MicroRNA Reverse Transcription kit (Takara Bio, Inc., Otsu, Japan) according to the manufacturer's protocol. To estimate the expression of miR-21, the quantification cycle (Cq) values were normalized using U6 as an internal control. The PCR results were separated by $2 \%$ agarose electrophoresis gel containing ethidium bromide $(0.5 \mu \mathrm{g} / \mathrm{ml})$ using electrophoresis apparatus (Bulletin \#M1704498; Bio-Rad Laboratories, Inc., Hercules, CA, USA) and a Visualizer (Bio-Rad GelDoc XR; Bio-Rad Laboratories, Inc.). For the analysis of PTEN and PDCD4 expression, complementary DNA was synthesized using PrimeScript $^{\mathrm{TM}}$ RT Master Mix (Takara Bio, Inc.) according to the manufacturer's protocol. RT-qPCR was carried out using a SYBR Premix Ex Taq ${ }^{\text {TI }}$ II (Takara Bio, Inc.). The housekeeping gene GAPDH was used for normalization. Primers are illustrated in Table II.

RT-qPCR was performed using the Rotor-Gene $3000^{\text {TM }}$ system with Rotor Gene Detection software (version 6.1.90; both from Qiagen, Inc., Valencia, CA, USA). The following thermocycling conditions were performed: $3 \mathrm{~min}$ at $95^{\circ} \mathrm{C}$; and 40 cycles of $95^{\circ} \mathrm{C}$ for $15 \mathrm{sec}, 58^{\circ} \mathrm{C}$ for $30 \mathrm{sec}$ and $72^{\circ} \mathrm{C}$ for $30 \mathrm{sec}$. All reactions were performed in triplicate. The $2^{-\Delta \Delta \mathrm{Cq}}$ method (13) was used for the relative quantification of the gene expression of miR-21, PTEN and PDCD4.

Western blot (WB) analysis. In order to extract the cellular protein, cells were lysed on ice for $30 \mathrm{~min}$ with radioimmunoprecipitation assay buffer $(50 \mathrm{mmol} / \mathrm{l}$ Tris- $\mathrm{HCl}, \mathrm{pH} 7.5$; $150 \mathrm{mmol} / \mathrm{l} \mathrm{NaCl} ; 0.5 \%$ deoxycholate; and $0.1 \%$ SDS). Protein concentration was determined using a Pierce ${ }^{\mathrm{TM}}$ BCA Protein Assay kit (Thermo Fisher Scientific Inc.) according to the manufacturer's protocol. Following denaturation in boiling water for 5 min, 20- $\mu \mathrm{g}$ samples were separated on 10\% SDS-PAGE gels (Bio-Rad Laboratories, Inc.) and transferred onto polyvinylidene fluoride membranes (EMD Millipore, Billerica, MA, USA).

The membranes were blocked using 5\% non-fat milk and incubated overnight at $4^{\circ} \mathrm{C}$ with primary rabbit polyclonal anti-human antibodies directed against PTEN (\#9188), PDCD4 (\#9535) or caspase-3 (\#9662; all primary antibody dilutions were 1:1,500; Cell Signaling Technology, Inc., Danvers, MA, USA). Membranes were incubated for $2 \mathrm{~h}$ at room temperature with a goat anti-rabbit horseradish peroxidase-conjugated immunoglobulin Ge secondary antibody (\#W10809; dilution, 1:3,000; Pierce; Thermo Fisher Scientific, Inc.). Subsequently, proteins were visualized using an ECL substrate (Immobilon Western Chemiluminescent HRP substrate; EMD Millipore) according to the manufacturer's protocol, using a Bio-Rad Molecular Imager ChemiDoc ${ }^{\mathrm{TM}}$ XRS+ with Image $\mathrm{Lab}^{\mathrm{TM}}$ software 2.0 (Bio-Rad Laboratories, Inc.) and analyzed by ImageJ (version 2.1.4.7; National Institutes of Health, Bethesda, MD, USA). Membranes were also probed with an anti-human antibody directed against GAPDH (1:10,000; Shanghai Kangcheng Biological Engineering Co., Ltd., Shanghai, China) to ensure equal loading of protein. All experiments were performed in triplicate.

Cell proliferation. Cell proliferation was measured using a cell counting kit (CCK-8; Dojindo Molecular Technologies, Inc., Kumamoto, Japan). Cells were seeded into 96-well plates $\left(1 \times 10^{3}\right.$ cells/well) and cultured for $24 \mathrm{~h}$. The viability of SK-N-SH cells transfected with miR-21 inhibitor or NC was 
Table I. Sequences of miR-21 inhibitor and control oligonucleotides, and primers used in their construction.

\begin{tabular}{cl}
\hline Oligonucleotide & \multicolumn{1}{c}{ Sequence } \\
\hline miR-21 inhibitor & 5'-TCAACATCAGTCTGATAAGCTA-3' \\
Forward, BamHI & 5'-GATCCTCAACATCAGTCTGATAAGCTACGATTCAACATCAGTCTGATAAGCTAACCGGTTCAA \\
& CATCAGTCTGATAAGCTATCACTCAACATCAGTCTGATAAGCTATTTTTGAATT-3' \\
Reverse, EcoRI & 5'-ACCGGTTAGCTTATCAGACTGATGTTGAATCGTAGCTTATCAGACTGATGTTGAGAATTCAAA \\
& AAATAGCTTATCAGACTGATGTTGAGTGATAGCTTATCAGACTGATGTTGA-3' \\
miR-NC & 5'-TTCTCCGAACGTGTCACGT-3' \\
Forward, BamHI & 5'-GATCCGTTCTCCGAACGTGTCACGTTTCAAGAGAACGTGACACGTTCGGAGAACTTTTTTG-3' \\
Reverse, EcoRI & 5'-AATTCAAAAAAGTTCTCCGAACGTGTCACGTTCTCTTGAAACGTGACACGTTCGGAGAACG-3' \\
\hline
\end{tabular}

miR, microRNA; NC, negative control.

Table II. Primer sequences used in quantitative polymerase chain reactions.

\begin{tabular}{lc}
\hline Primer & Sequence \\
\hline U6 & \\
Forward & 5'-ATTGGAACGATACAGAGAAGATT-3' \\
Reverse & 5'-GGAACGCTTCACGAATTTG-3' \\
miR-21 & \\
Forward & 5'-ACGTTGTGTAGCTTATCAGACTG-3' \\
Reverse & 5'-AATGGTTGTTCTCCACACTCTC-3' \\
GAPDH & \\
Forward & 5'-GAGTCAACGGATTTGGTCGT-3' \\
Reverse & 5'-TTGATTTTGGAGGGATCTCG-3' \\
PTEN & \\
Forward & 5'-GCACTGTTGTTTCACAAGATGATG-3' \\
Reverse & 5'-GCAGACCACAAACTGAGGATTG-3' \\
PDCD4 & \\
Forward & 5'-CGACAGTGGGAGTGACGCCCTTA-3' \\
Reverse & 5'-CAGACACCTTTGCCTCCTGCACC-3'
\end{tabular}

miR, microRNA; PDCD4, programmed cell death 4; PTEN, phosphatase and tensin homologue.

analyzed every $24 \mathrm{~h}$ following transfection. CCK-8 (10 $\mu \mathrm{l})$ was added to each well of the 96 -well plates and incubated in $37^{\circ} \mathrm{C}$ for $4 \mathrm{~h}$. Proliferation rates were determined at 0, 24, 48 and $72 \mathrm{~h}$ following transfection. The optical density was measured at wavelength of $490 \mathrm{~nm}$ using a 2550 EIA reader (Bio-Rad Laboratories, Inc.).

Hoechst 33342 staining. The SK-N-SH, SH-SY5Y and BE2C cell lines were cultured in 6-well tissue culture plates. The culture medium was removed and the cells were fixed in $4 \%$ paraformaldehyde for $10 \mathrm{~min}$ at room temperature. Following washing twice in PBS, the cells were stained with $10 \mu \mathrm{M}$ Hoechst 33342 (Sigma-Aldrich; Merck KGaA, Darmstadt, Germany) at $37^{\circ} \mathrm{C}$ for $30 \mathrm{~min}$. The nuclear structure of the cells was examined using an IX71 fluorescence microscope (Olympus Corporation, Tokyo, Japan). Quantitative analysis was performed by counting green fluorescent (apoptosis-positive) cells under $\mathrm{x} 400$ magnification in three independent fields. The values are expressed as the percentage of apoptotic cells relative to the total number of cells per field.

Statistical analysis. All data are presented as the mean \pm standard deviation. Statistical analysis was performed using SPSS statistical software (version 17.0; SPSS, Inc., Chicago, IL, USA). The significance of differences between groups was analyzed using one-way analysis of variance. $\mathrm{P}<0.05$ was considered to indicate a statistically significant difference.

\section{Results}

miR-21 inhibitor downregulates miR-21 in SK-N-SH cells. The infection efficiency of lentivirus in SK-N-SH, SH-SY5Y and BE2C cells was estimated at 95, 75 and 95\%, respectively through fluorescence microscopy (Fig. 1A). The expression of miR-21 was detected in all three cell lines. The results of $2 \%$ agarose gel electrophoresis demonstrated that the size of the PCR products was correct (U6, $70 \mathrm{bp}$; miR-21, $82 \mathrm{bp}$ ), without any non-specific bands, and that only specifically amplified products were present (Fig. 1B).

Statistical analysis demonstrated no significant difference between miR-21 expression in SK-N-SH and SH-SY5Y cells (Fig. 1C). However, miR-21 expression in BE2C cells was significantly lower compared with that in SK-N-SH and SH-SY5Y cell lines (both $\mathrm{P}<0.01$; Fig. 1C). Based on its miR-21 expression and infection efficiency, the SK-N-SH cell line was selected for further experiments. Following transfection, the expression of miR-21 was determined by RT-qPCR. The expression of miR-21 was significantly diminished in LV3-miR-21 inhibitor-transfected cells (1381) compared with that in untransfected SK-N-SH and LV3-NC-transfected cells (both P<0.01; Fig. 1D).

miR-21 inhibitor upregulates messenger RNA (mRNA) and protein expression of PTEN and PDCD4 in 1381 cells. Following miR-21 inhibitor transfection, the expression of PTEN and PDCD4 protein in 1381 cells was increased compared with that in the control SK-N-SH and NC groups, in which PTEN and PDCD4 expression was similar (Fig. 2A). Quantification of triplicate WB analysis demonstrated a significant increase in the expression of PTEN (Fig. 2B) and 
A
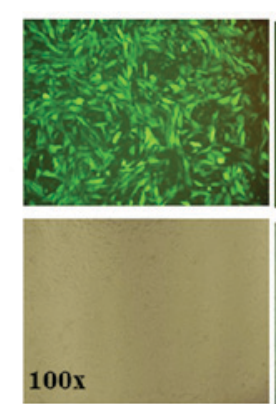

SK-N-SH $95 \%$
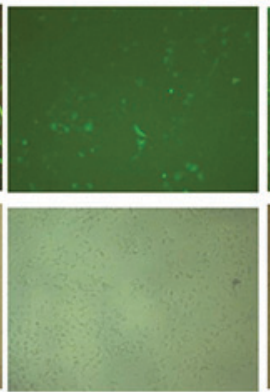

SH-SY5Y $75 \%$
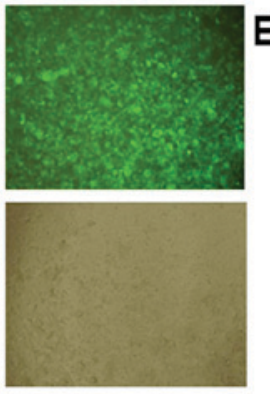

BE2C $95 \%$
B
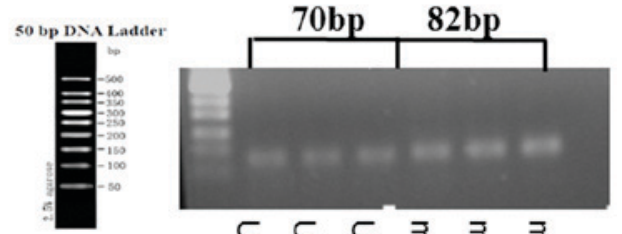

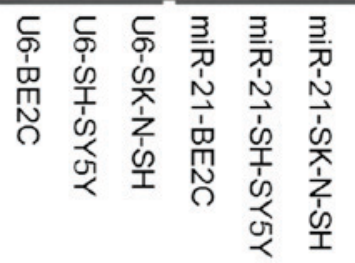

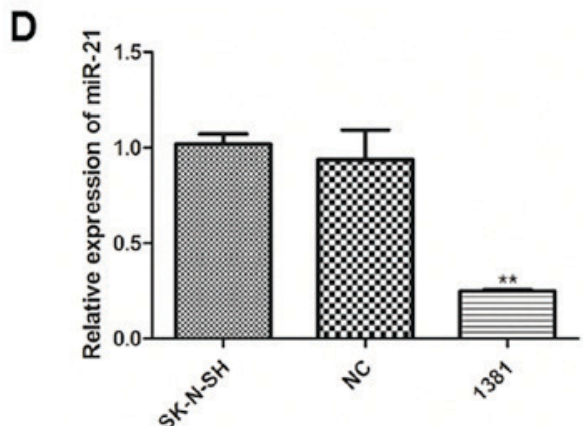

Figure 1. Transfection of NB cell lines with miR-21 inhibitory or NC oligonucleotides. (A) Infection efficiency of lentivirus determined by fluorescence microscopy in SK-N-SH (95\%), SH-SY5Y (75\%) and BE2C (95\%) cells (x100). The top panels are fluorescent images and the bottom panels are white light images of the cells. (B) Gel electrophoresis of PCR products illustrating specific amplification of correctly-sized miR-21 products in each cell line. (C) RT-qPCR analysis demonstrated no significant difference between miR-21 expression relative to U6 in SK-N-SH and SH-SY5Y cells. However, significantly decreased expression of miR-21 was observed in BE2C cells compared with that in SK-N-SH and SH-SY5Y cells. (D) RT-qPCR analysis demonstrated significantly decreased expression of miR-21 relative to U6 in 1381 cells compared with that in untransfected SK-N-SH cells and cells transfected with NC. ${ }^{* *} \mathrm{P}<0.01$ vs. NC and SK-N-SH. NC, negative control; miR, microRNA; 1381, SK-N-SH cells transfected with miR-21 inhibitor; RT-qPCR, reverse transcription-quantitative polymerase chain reaction.

A

PTEN

PDCD4

GAPDH

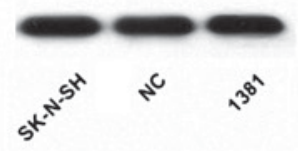

B

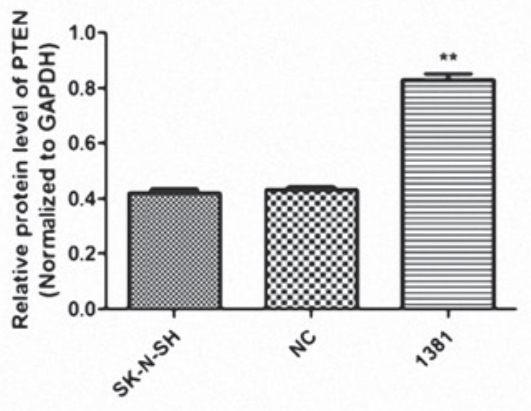

$\mathrm{C}$ \&

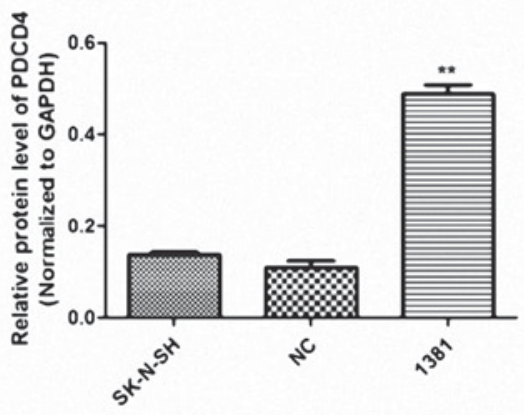

D

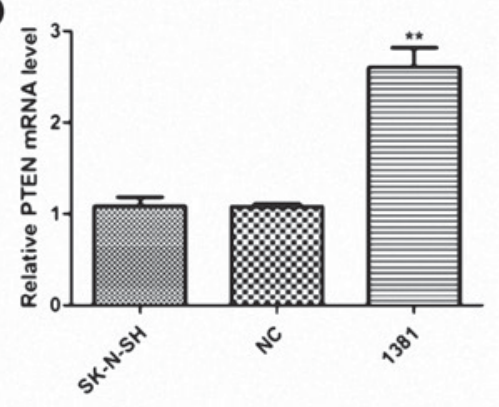

$\mathbf{E}$

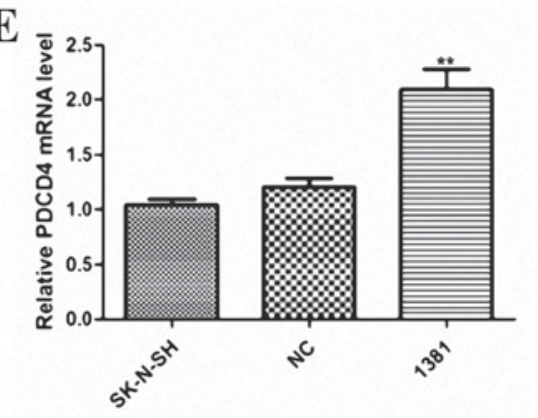

Figure 2. Expression of PTEN and PDCD4 protein and mRNA in 1381 cells transfected with a miR-21 inhibitory oligonucleotide. (A) Representative WB analysis of protein expression in untransfected SK-N-SH, NC-transfected and 1381 cells (left to right lanes, respectively). Quantitation of triplicate WB analysis demonstrated that expression of (B) PTEN and (C) PDCD4 was significantly increased when miR-21 was inhibited in 1381 cells. RT-qPCR analysis of (D) PTEN and (E) PDCD4 mRNA expression relative to U6 demonstrated a significant increase in 1381 cells compared with that in untransfected SK-N-SH cells and cells transfected with NC. ${ }^{* *} \mathrm{P}<0.01$ vs. NC and SK-N-SH. NC, negative control; miR, microRNA; 1381, SK-N-SH cells transfected with miR-21 inhibitor; RT-qPCR, reverse transcription-quantitative polymerase chain reaction; WB, western blot; PDCD4, programmed cell death 4; PTEN, phosphatase and tensin homologue; mRNA, messenger RNA. 


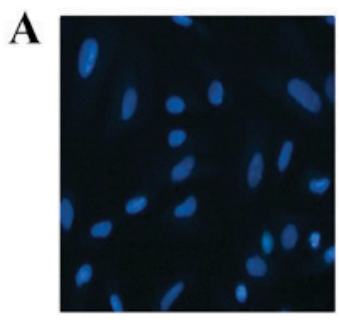

SK-N-SH

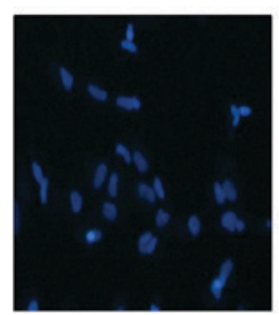

NC

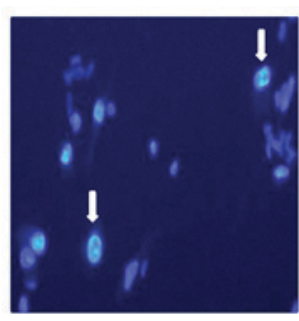

1381
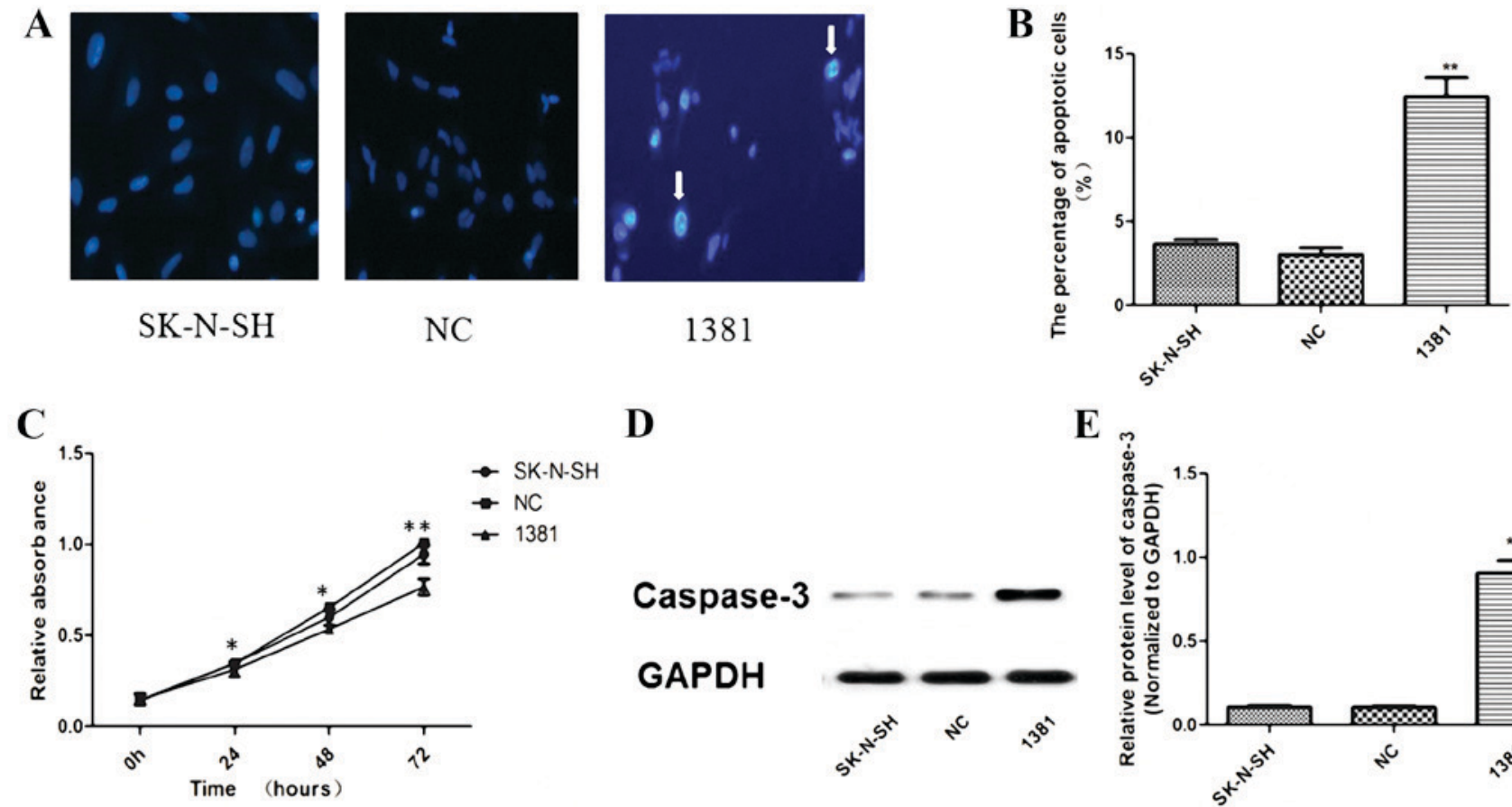

E

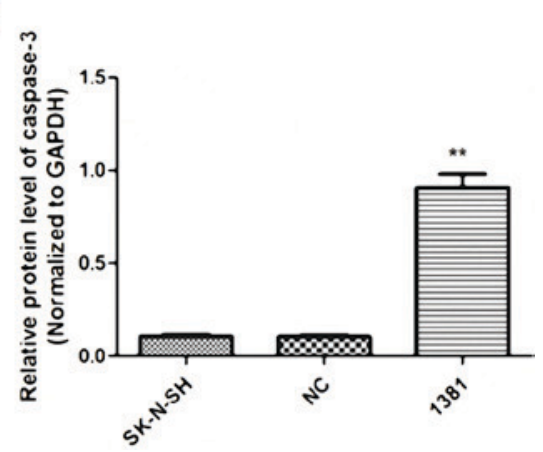

Figure 3. Apoptosis, proliferation and caspase-3 activation in 1381 cells. (A) Hoechst 33342 nucleic acid staining in untransfected SK-N-SH, NC-transfected and 1381 cells. Arrows indicate the characteristic appearance of nuclear chromatin condensation and nuclear fragmentation in apoptotic 1381 cells. Magnification, $\mathrm{x} 400$. (B) The percentage of apoptotic cells in the 1381 group was significantly increased compared with that in $\mathrm{NC}$ and untransfected $\mathrm{SK}-\mathrm{N}-\mathrm{SH}$ cells $\left({ }^{* *} \mathrm{P}<0.01\right)$. (C) CCK-8 assay demonstrated that the miR-21-inhibitor significantly reduced the proliferation of 1381 cells compared with the viability of SK-N-SH and NC cells at 24, 48 and $72 \mathrm{~h}$. WB analysis illustrating a (D) representative image from triplicate experiments and (E) quantitative results, indicating a significantly increased level of activated caspase-3 protein when miR-21 was inhibited in 1381 cells, compared with that in NC and $\mathrm{SK}-\mathrm{N}-\mathrm{SH}$ cells. ${ }^{*} \mathrm{P}<0.05$, ${ }^{* *} \mathrm{P}<0.01$ vs. NC and SK-N-SH. NC, negative control; miR, microRNA; 1381, SK-N-SH cells transfected with miR-21 inhibitor; WB, western blot; CCK, cell counting kit.

PDCD4 (Fig. 2C) protein compared with that in the NC and SK-N-SH groups (both $\mathrm{P}<0.01$ ). In addition, RT-qPCR analysis revealed significantly increased expression of PTEN (Fig. 2D) and PDCD4 (Fig. 2E) mRNA in 1381 cells compared with that in the control cell group (both $\mathrm{P}<0.01$ ).

miR-21 inhibitor reduces proliferation and induces apoptosis in 1381 cells. Hoechst 33342 nucleic acid staining was performed on untransfected cells and cells transfected with LV3-NC and LV3-miR-21 inhibitor. Fig. 3A illustrates the characteristic appearance, including nuclear chromatin condensation and nuclear fragmentation, in apoptotic 1381 cells. Transfection with the miR-21 inhibitor resulted in a significant increase in the number of apoptotic 1381 cells $(12.45 \pm 1.99 \%)$ compared with the basal apoptosis level observed in the NC $(3.04 \pm 0.68 \%)$ and untransfected SK-N-SH $(3.65 \pm 0.46 \%)$ groups (both $\mathrm{P}<0.01$; Fig. 3B). The CCK-8 assay revealed that transfection with the miR-21 inhibitor significantly inhibited the proliferation of 1381 cells compared with the cell viability noticed in the untransfected SK-N-SH and NC groups ( $\mathrm{P}<0.05$; Fig. 3C). Furthermore, WB analysis (Fig. 3D) demonstrated a significant increase in the expression of activated caspase-3 protein in 1381 cells compared with that in NC and SK-N-SH cells $(\mathrm{P}<0.01$; Fig. 3E).

\section{Discussion}

miRNAs are endogenous, 19-22-nucleotide long, non-coding RNAs that can negatively regulate protein expression by inducing the degradation of target mRNAs, inhibiting their translation or both, by specifically binding to the 3 ' untranslated regions of target mRNAs (4). Although increasing evidence suggests that a novel class of miRNAs can regulate various target genes, including oncogenes and tumor suppressors, the role of miRNAs in NB remains unclear (14).

Previous evidence indicates that miR-21 participates in the development and progression of various types of human tumors, including glioblastoma, hepatocellular, lung, colon, and prostate cancer $(15,16)$. miR-21 is encoded by chromosome 17q23.2, which is frequently involved in unbalanced translocations in NB cell lines (15). In addition, miR-21 is among the most frequently detected miRNAs in primary NB tumors and NB cell lines $(17,18)$. Furthermore, NB cell lines that have been established from human NB cells exhibit similar cellular heterogeneity. Based on the morphological appearance, biochemical properties and growth patterns, three major cell types have been identified in NB cell lines. These have been designated as neuroblastic, substrate-adherent and non-neuronal, and intermediate-type NB cells (19). SH-SY5Y, SK-N-SH, and BE2C cell lines represent the above three types, respectively (19). The SK-N-SH cell line was selected following consideration of its miR-21 expression and the infection efficiency of the lentivirus into the target cells.

PTEN and PDCD4 are target genes of miR-21 (20), which were validated by the three following target prediction programs: PicTar (http://pictar.mdc-berlin.de/), TargetScan (http://www.targetscan.org) and miRanda (http://www. microrna.org). PDCD4 suppresses several proteins that regulate translation and cell proliferation, and has been implicated in tissue invasion and proliferation $(20,21)$. PTEN is a phosphatase 
that maintains low levels of $\mathrm{PIP}_{3}$ by conversion to phosphatidylinositol 4,5-bisphosphate. When PTEN fails to maintain this homeostasis, $\mathrm{PIP}_{3}$ levels increase and activate the AKT serine/threonine kinase (Akt) signaling pathway. Activation of the Akt signaling pathway leads to several effects, including promotion of cell growth and proliferation, and inhibition of apoptosis $(20,21)$. For example, miR-21 promotes cell growth and migration by targeting PDCD4 gene expression in Kazakh esophageal squamous cell carcinoma (22). Similarly, overexpression of miR-21 in cervical cancer promotes the proliferation and migration of cells via inhibition of PTEN (23). The results of the present study have demonstrated that the downregulation of miR-21 following transfection with a miR-21-inhibitor results in a significant increase in PTEN and PDCD4 mRNA and protein expression compared with that observed in untransfected and NC-transfected cells. Furthermore, miR-21 inhibitor transfection led to a significant reduction in tumor cell growth and induction of apoptosis compared with the effects observed in untransfected and NC-transfected cells. Thus, this result suggests that the reduction of miR-21 activates the caspase- 3 signaling pathway, possibly mediated by PTEN/PDCD4 induction, to subsequently inhibit cell proliferation and induce apoptosis.

Chan et al (24) demonstrated that miR-21 is commonly and markedly upregulated in human glioblastoma, and that inhibiting miR-21 expression leads to caspase-3/caspase-7 activation and associated apoptotic cell death in multiple glioblastoma cell lines. Zhou et al (25) reported that the reduction of miR-21 by antisense oligonucleotides activates the caspase- 9 and caspase- 3 signaling pathways, possibly mediated by multiple potential target genes, and subsequently induce glioma cell apoptosis. Recently, White et al (26) demonstrated that endothelial apoptosis in pulmonary hypertension is controlled by the miR-21/PDCD4/caspase-3 axis. Li et al (27) reported that, in ovarian cancer A2780 cells, icariin substantially decreased miR-21 expression, increased the expression levels of target proteins PTEN and reversion-inducing-cysteine-rich protein with kazal motifs, suppressed cell proliferation, accelerated apoptosis and increased caspase-3 activity, compared with the effects observed in the untreated control group. The results of the current study indicate that miR-21 regulates the potential targets PTEN/PDCD4 to activate the caspase-3 signaling pathway. However, the mechanism underlying miR-21-mediated regulation of the caspase-3 signaling pathway remains unclear and warrants further investigation.

In conclusion, the present study has demonstrated that miR-21 expression is downregulated in NB cells, and has revealed that the inhibition of miR-21 can promote cell apoptosis and inhibit proliferation by upregulating tumor-suppressive PTEN/PDCD4 expression via caspase-3 activation. To the best of our knowledge, the present study is the first to confirm that miR-21 regulates PTEN/PDCD4 in NB. These results suggest that miR-21 is an effective therapeutic target in the treatment of patients with NB.

\section{Acknowledgements}

The present study was supported by the Shanghai Committee of Science and Technology (grant nos. 15411961900 and 12431900205, awarded to Professor Kai Li and Dr Xiaolong
Zhao, Department of Endocrinology, Huashan Hospital of Fudan University, Shanghai, China, respectively).

\section{References}

1. Sharp SE, Gelfand MJ and Shulkin BL: Pediatrics: Diagnosis of neuroblastoma. Semin Nucl Med 41: 345-353, 2011.

2. Maris JM: Recent advances in neuroblastoma. N Engl J Med 362: 2202-2211, 2010.

3. Zhu H, Zheng J, Xiao X, Zheng S, Dong K, Liu J and Wang Y: Environmental endocrine disruptors promote invasion and metastasis of SK-N-SH human neuroblastoma cells. Oncol Rep 23: 129-139, 2010.

4. Bartel DP: MicroRNAs: Genomics, biogenesis, mechanism, and function. Cell 116: 281-297, 2004.

5. Cheng L, Yang T, Kuang Y, Kong B, Yu S, Shu H, Zhou H and Gu J: MicroRNA-23a promotes neuroblastoma cell metastasis by targeting CDH1. Oncol Lett 7: 839-845, 2014.

6. Chen X, Pan M, Han L, Lu H, Hao X and Dong Q: miR-338-3p suppresses neuroblastoma proliferation, invasion and migration through targeting PREX2a. FEBS Lett 587: 3729-3737, 2013.

7. Wu K, Yang L, Chen J, Zhao H, Wang J, Xu S and Huang Z: miR-362-5p inhibits proliferation and migration of neuroblastoma cells by targeting phosphatidylinositol 3-kinase-C2 $\beta$. FEBS Lett 589: 1911-1919, 2015.

8. Zhang H, Pu J, Qi T, Qi M, Yang C, Li S, Huang K, Zheng L and Tong Q: MicroRNA-145 inhibits the growth, invasion, metastasis and angiogenesis of neuroblastoma cells through targeting hypoxia-inducible factor 2 alpha. Oncogene 33: 387-397, 2014.

9. Wang G, Wang JJ, Tang HM and To ST: Targeting strategies on miRNA-21 and PDCD4 for glioblastoma. Arch Biochem Biophys 580: 64-74, 2015.

10. Qi L, Bart J, Tan LP, Platteel I, Sluis Tv, Huitema S, Harms G, Fu L, Hollema $\mathrm{H}$ and Berg Av: Expression of miR-21 and its targets (PTEN, PDCD4, TM1) in flat epithelial atypia of the breast in relation to ductal carcinoma in situ and invasive carcinoma. BMC Cancer 9: 163, 2009.

11. Asangani IA, Rasheed SA, Nikolova DA, Leupold JH, Colburn NH, Post S and Allgayer H: MicroRNA-21 (miR-21) post-transcriptionally downregulates tumor suppressor Pdcd4 and stimulates invasion, intravasation and metastasis in colorectal cancer. Oncogene 27: 2128-2136, 2008.

12. Zhang BG, Li JF, Yu BQ, Zhu ZG, Liu BY and Yan M: microRNA-21 promotes tumor proliferation and invasion in gastric cancer by targeting PTEN. Oncol Rep 27: 1019-1026, 2012.

13. Livak KJ and Schmittgen TD: Analysis of relative gene expression data using real-time quantitative PCR and the 2(-Delta Delta C(T)) method. Methods 25: 402-408, 2001.

14. Gentilin E, Degli Uberti E and Zatelli MC: Strategies to use microRNAs as therapeutic targets. Best Pract Res Clin Endocrinol Metab 30: 629-639, 2016.

15. Krichevsky AM and Gabriely G: miR-21: A small multi-faceted RNA. J Cell Mol Med 13: 39-53, 2009.

16. Pfeffer SR, Yang CH and Pfeffer LM: The role of miR-21 in cancer. Drug Dev Res 76: 270-277, 2015.

17. Afanasyeva EA, Hotz-Wagenblatt A, Glatting KH and Westermann F: New miRNAs cloned from neuroblastoma. BMC Genomics 9: 52, 2008.

18. Buechner J, Henriksen JR, Haug BH, Tomte E, Flaegstad T and Einvik C: Inhibition of mir-21, which is up-regulated during MYCN knockdown-mediated differentiation, does not prevent differentiation of neuroblastoma cells. Differentiation 81: 25-34, 2011.

19. Yang S, Zheng J, Xiao X, Xu T, Tang W, Zhu H, Yang L, Zheng S, Dong K, Zhou G and Wang Y: SOX2 promotes tumorigenicity and inhibits the differentiation of I-type neuroblastoma cells. Int J Oncol 46: 317-323, 2015.

20. Buscaglia LE and Li Y: Apoptosis and the target genes of microRNA-21. Chin J Cancer 30: 371-380, 2011.

21. Li X, Huang K and Yu J: Inhibition of microRNA-21 upregulates the expression of programmed cell death 4 and phosphatase tensin homologue in the A431 squamous cell carcinoma cell line. Oncol Lett 8: 203-207, 2014.

22. Liu T, Liu Q, Zheng S, Gao X, Lu M, Yang C, Dai F, Sheyhidin I and $\mathrm{Lu} \mathrm{X}$ : MicroRNA-21 promotes cell growth and migration by targeting programmed cell death 4 gene in Kazakh's esophageal squamous cell carcinoma. Dis Markers 2014: 232837, 2014. 
23. Xu J, Zhang W, Lv Q and Zhu D: Overexpression of miR-21 promotes the proliferation and migration of cervical cancer cells via the inhibition of PTEN. Oncol Rep 33: 3108-3116, 2015.

24. Chan JA, Krichevsky AM and Kosik KS: MicroRNA-21 is an antiapoptotic factor in human glioblastoma cells. Cancer Res 65 : 6029-6033, 2005.

25. Zhou X, Zhang J, Jia Q, Ren Y, Wang Y, Shi L, Liu N, Wang G, $\mathrm{Pu}$ P, You Y and Kang C: Reduction of miR-21 induces glioma cell apoptosis via activating caspase 9 and 3. Oncol Rep 24: 195-201, 2010 .
26. White K, Dempsie Y, Caruso P, Wallace E, McDonald RA, Stevens H, Hatley ME, Van Rooij E, Morrell NW, MacLean MR and Baker AH: Endothelial apoptosis in pulmonary hypertension is controlled by a microRNA/programmed cell death 4/caspase-3 axis. Hypertension 64: 185-194, 2014.

27. Li J, Jiang $\mathrm{K}$ and Zhao F: Icariin regulates the proliferation and apoptosis of human ovarian cancer cells through microRNA-21 by targeting PTEN, RECK and Bcl-2. Oncol Rep 33: 2829-2836, 2015. 